\title{
Synthesen unter Einwirkung von Zinkchlorid in der hydroaromatischen Reihe; \\ von
}

\section{J. Kondakow.}

(Aus dem Laboratorium des pharmaceutischen Instituts zu Dorpat.)

Durch meine Untersuchungen über die Synthese in der Olefinreihe wurde festgestellt, dass die Aethylenkohlenwasserstoffe, welche ein tertiäres Kohlenstoffatom bei der Doppelbindung haben, sich in Gegenwart von Zinkchlorid mit organischen Säuren verbinden und Ester bilden; wenn dieselben Chloranhydride tertiärer Alkohole addiren, so bilden sie Chloranhydride tertiärer Alkohole von höherer Zusammengesetztheit.

Dieses Verhalten der Olefine verfolgend, sprach ich damals die Voraussetzung aus, dass „... das Zinkchlorid zur Entdeckung des tertiären Kohlenstoffatoms bei der Doppelbindung dienen kann ...."

Auf diese Zulassung gestützt, konnte man erwarten, dass die hydroaromatischen Kohlenwasserstoffe, welche eine Doppelbindung im Ringe, und noch vielmehr die, welche eine solche in der Seitenkette haben, in Betracht ihrer zweifellosen Aehnlicheit mit den Olefinen, in ähnlicher Weise unter oben angeführter Bedingung reagiren werden.

Aus der Zahl der Kohlenwasserstoffe der ersten Gruppe wurden in ihrem Verhalten zu der erwähnten Reaction das Menthomenthen und Carvomenthen geprüft, es sind dieses Kohlenwasserstoffe, welche fraglos die Doppelbindung im Ringe haben und in ihrer grössten Menge aus Isomeren mit dem tertiären Kohlenstoffatom bei der Doppelbindung bestehen.

Eine geringe Beimengung im Carvomenthen vom Isomeren mit der Doppelbindung bei dem secundären Kohlenwasserstoffatom ist zweifellos nach den Untersuchungen von Kondakow und Lutschinin ${ }^{1}$ ) vorhanden, welche die Nicht-Einheitlichkeit dieses Kohlenwasserstoffs nachgewiesen haben.

1) Dies. Journ. [2] 60, 274-277. 
202 Kondakow: Synthesen unter Einw. v. Zinkchlorld etc.

Was die von Wagner ${ }^{1}$ ) gefolgerte Beimengung des Kohlenwasserstoffs von derselben Constitution im Menthomenthen anbelangt, so findet diese Folgerung weder eine Bestätigung durch positive Thatsachen, noch durch die Untersuchung von Tolloczkoº ${ }^{2}$, endlich auch nicht durch den ungewöhnlich constanten Siedepunkt des Menthomenthens. ${ }^{3}$ )

Diese Folgerung wird bis jetzt nur dadurch gerechtfertigt, dass das Menthylchlorid, aus welchem gewöhnlich das Menthomenthen erhalten wird, als Gemisch zweier Chloride erscheint, wie es zuerst Kondakow ${ }^{4}$ ) zeigte und wie es in der Folge durch Slawinsky ${ }^{5}$ ) und Kursan off $f^{6}$ ) bestätigt wurde, welche in genügend reiner Form das secundäre Menthylchlorid abgeschieden haben, das im Uebrigen vor ihnen von Berkenheim ?) erhalten war. Solch' ein Gemenge von Chlo. riden kann aber beim Abspalten von Chlorwasserstoff nach der bekannten Gesetzmässigkeit zwei Menthomenthene geben, von denen das eine das gleiche mit einem der Isomeren des Carvomenthens sein wird. Folglich wird das Menthomenthen und das Carvomenthen aus einem Gemisch folgender dreier Kohlenwasserstoffe bestehen, von welchem das Isomere II so-

) Ber. 26, 2270 .

$\left.{ }^{2}\right)$ Journ. russ. chem. Ges. 29, 39. Das Menthen von Tolloczko scheint mit Menthan verunreinigt gewesen zu sein.

${ }^{3}$ ) Es stehen noch Isomere mit einer Doppelbindung in der Seitenkette zu erwarten, $\Delta, \Delta_{4} ;$ durch mein Verfabren können sie nicht entdeckt werden.

4) Ber. 28, 1618 (1895). Kursa n off stellt in seinen Abhandlungen die Geschichte dieser Frage nicht in der Reihenfolge dar. Nach Berkenheim zeigte vor den anderen Kondakow, dass das Menthylchlorid aus einem Gemisch von Chloriden besteht, in welchem das eine tertiäre von Alkalien leichter, das andere secundäre schwerer zerlegt wird. Derselbe constatirte es in der Folge auch bei den Chloranhydriden, Bromanhydriden und Jodanhydriden der anderen Alkohole und nahm sie als Allgemeinregel an. Daher irrt sich Kursan off, wenn er sagt: „Kondak off und Lutschinin sind der Ansicht, dass bei der Einwirkung ron Jodwasserstoffsäure auf Menthol Isomerisation bei gewöhnlicher Temperatur stattfindet und kein secundäres', sondern ein tertiäres Jodid entsteht."

5) Journ. russ. chem. Ges. 29, 118.

$\left.{ }^{6}\right)$ Das. 33, 289 (2); Ann. Chem. 318, 327.

7) Ber. 25, 686; Journ. russ. chem. Ges. 24, 179. 
Kondakow: Synthesen unter Einw. v. Zinkehlorid etc. 203 wohl im Menthomenthen, als auch im Carvomenthen in geringer Beimengung enthalten ist.

I.<smiles>CC1CCC(C(C)C)CC1</smiles>

II.<smiles>[14CH3]</smiles><smiles>[CH]C</smiles><smiles></smiles><smiles>CCCCC</smiles><smiles>C[CH]C</smiles>

III.<smiles>C=C</smiles><smiles>CCC</smiles><smiles>CCC</smiles>

(

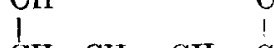<smiles>CC</smiles>

Versuche, zu diesen Menthenen unter Einwirkung ron Zinkchlorid Essigsäure zu addiren, verliefen resultatlos, weitere Versuche, das Menthylchlorid zum Menthomenthen unter gleichen Bedingungen zu addiren, hatten nur theilweisen Erfolg. So gab ein Gemisch von 30,0 Grm. Menthen und 40,0 Grm. Menthylchlorid, welchem 4 Grm. Zinkchlorid hinzugefügt waren nachdem es fast $3 \mathrm{Jahre}$ im eingeschmolzenen Rohre gelegen hatte, annähernd $30,0 \mathrm{Grm}$. Dimenthen mit Chlorwasserstoffdimenthen.

In analoger Weise addirt sich das tertiäre Menthylchlorid (aus Menthen und Chlorwasserstoff) zum Trimethyläthylen und giebt ein tertiäres Chlorid, obgleich dessen Constitution noch nicht bestätigt ist, aber nach bekannten Analogen in der Fettreihe zu schliessen, von folgendem Bau:<smiles>CC(C)C1CCC(C)C(C(C)C)C1</smiles>

Zur weiteren Prüfung der angeführten Behauptung wurden in den Kreis unserer Untersuchungen noch andere hydroaromatische Kohlenwasserstoffe hineingezogen, erstens solche, 
204 Kondakow: Synthesen unter Einw. v. Zinkchlorid ete.

welche eine Doppelbindung bei dem secundär-secundären Kohlenstoffatom im Ringe haben, dann zweitens solche, welche ausser der Doppelbindung im Ringe zwischen den secundärsecundären oder secundär-tertiären Kohlenstoffatomen noch eine Diagonalbindung enthalten, drittens auch noch solche, die mit oder ohne Diagonalbindung noch eine Doppelbindung in der Seitenkette besitzen.

Aus der Zahl der Kohlenwasserstoffe der ersten Gruppe war von uns das Tetrahydrotoluol gewählt worden, welches uns zur Darstellung einer ganzen Reibe von Verbindungen diente, die in unserer Abhandlung ${ }^{1}$ ) beschrieben sind. Dieser Kohlenwasserstoff wie auch die Menthene verbinden sich überhaupt nicht mit organischen Säuren in Gegenwart von Zinkchlorid.

Aus der zweiten Gruppe der Kohlenwasserstoffe wählten wir das Camphen und Fenchen aus, Kohlenwasserstoffe, deren Constitution beim Anfange unserer Arbeit als völlig festgestellt galt, bei ihnen wurde die Gegenwart einer Doppelbindung im Ringe oder zwischen den bisecundären Kohlenstoffatomen (Camphen) oder zwischen den secundär-tertiären Kohlenstoffatomen (Fenchen) angenommen. Unsere Untersuchungen, welche gar nicht übereinstimmende Ergebnisse mit dieser Anschauung über die Constitution des Camphens und Fenchens gaben, waren schon im Mai vorigen Jahres fertig, zu meinem Bedauern konnten sie nicht rechtzeitig veröffentlicht werden, weil sich die Formalitäten mit dem deutschen Reichspatentamt verzögerten und wir dorthin eine Patentbeanspruchung eingeschickt hatten für die weiter unten zu beschreibenden Substanzen. In dieser Zeit, als sich die Patentformalitäten hinzogen, wurde die Frage über die Constitution des Camphens einer Durcharbeitung unterzogen und schritt dabei bemerkbar fort, und führte andere Forscher gerade zu analogen Schlüssen wie die unsrigen. Bis zur Veröffentlichung unserer Arbeit befand sich die Frage über die Constitution des Camphens und Fenchens in dieser nicht entschiedenen Lage.

3) Dies. Journ. [2]61, 477. 
Kondakow: Synthesen unter Einw. v. Zinkchlorid etc. 205

Ueber die Camphene.

Auf Grund der zahlreichen alten und neuen Untersuchungen über das Camphen wird angenommen, dass die Constitution desselben in directem Zusammenhang mit dem Bau des Camphers, des Borneols und in naher Beziehung mit dem des Pinens steht. Von den vielen, zu verschiedenen Zeiten vorgeschlagenen Formeln für den Campher richtete sich die Aufmerksamkeit der Forscher besonders auf die Formeln von Kekulé, Haller, Kanonnikoff, Tiemann und auf die zwei Formeln von Bredt; von letzteren wird gegenwärtig fast von allen Forschern die im Jahre 1893 vorgeschlagene Formel allgemein angenommen.

Der secundäre Alkohol, welcher bei der Reduction des Camphers erhalten wird (Berthelot, Baubigny, Haller, Kachler, Montgolfier, Jackson-Menokl, Immendorff, Wallach, Beckmann u. a.), wird das künstliche Borneol darstellen, das zur Darstellung der Camphene diente und noch dient.

Ihrerseits werden die Camphene, übergeführt in das Isoborneol und die Pinene in das Borneol oder Chlorwasserstoffpinen, welches jetzt als Bornylchlorid (?) angenommen wird, zur. Gewinnung desselben Camphens angewandt.

Nachdem Bredt ${ }^{1}$ ) den $B \backsim u$ für den Campher festgestelit hatte, schlug er für das Camphen die Formel<smiles>CCC(C)C(C)(C)C</smiles>

vor, welche von Niemand bestritten wurde, sogar nicht bis zur letzten Zeit, weil von Niemandem die Möglichkeit einer Isomerisation der für seine Darstellung gebrauchten Haloïdanhydride des Borneols vermuthet wurde; die Vermuthung wurde auch nach der Entdeckung und Untersuchung des von Bertram und Walbaum gefundenen, dem Borneol isomeren Alkohol Isoborneol ansgesprochen.

1) Ber. 1893, 3047. 
206 Kondakow: Synthesen unter Einw. v. Zinkchlorid etc.

Die Möglichkeit solcher Umwandlung in der Borneolreihe trat nur dann hervor, als es uns gelang Umwandlungen ähnlicher Art bei anderen secundären hydroaromatischen Alkoholen genau zu verfolgen.

Schon im Jahre 1895, als ich die Ergebnisse über die Umwandlung der Derivate des secundären Menthols in tertiäre veröffentlichte, wies ich auf die damals bekannten analogen Fälle in der Fettreihe hin, und sprach Folgendes in Bezug auf die secundären hydroaromatischen Alkohole aus: „Da dieses nun der Fall ist, liessen sich auch für andere ähnliche Fälle, statt secundärer, tertiäre Produkte erwarten. . ..."1) Dieselbe Voraussetzung wurde im Jahre 1897 in diesem Journal in der Bemerkung "Ueber einen neuen Fall von Isomerisation des Dihydrocarvons zu Carvenon" ausgesprochen.

Darauf wurde in der von mir zusammen mit Lutschinin am 15./27. Juni i 897 veröffentlichten Abhandlung, die oben angeführte Behauptung durch die Derivate des Carvomenthens definitiv bestätigt, wobei noch hinzugefügt war, dass dasselbe beim Fenchylalkohol zu erwarten sei, der dem Borneol so ähnlich ist. Alle diese unseren Annahmen wurden auf das beste durch unsere späteren Untersuchungen bewiesen. Folglich ist aus der oben angefübrten Hinweisung mit zweifelloser Klarheit ersichtlich, dass von uns nicht nur vor allen anderen die Isomerisation der Derivate einiger secundärer hydroaromatischer Alkohole zu tertiären festgesetzt wurde, sondern es wurde auch die Möglichkeit anderer analoger Fälle vorausgesagt, wie es auch später von uns und anderen Forschern bewiesen wurde.

Alles dieses gab uns Grund darauf zu rechnen, dass nach bestehender Gewohnheit das Untersuchungsvorrecht bei der weiteren Bearbeitung der Frage gesichert sei. Anderer Ansicht schienen Wagner und Brickner zu sein, als sie in Jahre 1899 ihre Arbeit unter dem Titel: „Ueber die Beziehung der Pinenhalö̈dhydrate zu den Halö̈danhydriden des Borneols" ${ }^{2}$ ) veröffentlichten, wo wir gerade unsere Untersuchung ausführten; diese Arbeit ist nicht nur ihrer Idee nach, sondern auch in vielen Einzelheiten unserer Arbeit analog
1) Ber. 28, 1621 .
") Das. 32, 2302. 
Kondakow: Synthesen unter Einw. v. Zinkchlorid etc. 207

ausgeführt. In ihr beschreiben Wagner und Brickner Ergebnisse, welche feststellen, dass die Haloüdanhydride des Borneols nicht dem Borneol - dem secundären Alkohol, sondern dem Isoborneol - dem tertiären Alkohol entsprechen, wobei sie nicht mit einem Worte verlauten, dass die von ihnen gewonnenen Thatsachen zu der Kategorie der von uns gefundenen gehören, die von uns veröffentlichten vervollständigen und die von uns zuerst ausgesprochene oben angeführte Behauptung bestätigen. Dass es sich so verhält, ist aus dem Vergleich unserer zweiten Abhandlung mit der von Wagner und Brickuer ersichtlich, welche letztere $1 \frac{1}{2}$ Monate später zum Druck eingelaufen ist als unsere. Dieses giebt uns das Recht zu hoffen, dass sowohl Wagner selbst, als auch die anderen Forscher für uns das Prioritätsrecht über die Feststellung der Isomerisation secundärer hydroaromatischer Alkohole zu tertiären anerkennen.

Weiter ist aus unseren Untersuchungen ersichtlich, dass die Produkte nach der Abspaltung der Halö̈dwasserstoffsäuren von den Haloïdanhydriden des Menthols, Carvomenthols, Fenchylalkohols erwiesenermaassen ihrer Haupttmenge aus den Derivaten der tertiären Alkohole bestehen, vorzïglich den letzteren, und nur in geringeren Maassen den Derivaten secundärer Alkohole entsprechen. Gleichzeitig wurde von uns gezeigt, dass die Haloïdanhydride secundärer Alkohole bedeutend schwerer durch Alkalien, wie Anilin, Chinolin und alkoholische Kalilauge zerlegt werden. Nachdem wir die Isomerisation der Derivate secundärer Alkohole zu tertiären Derivaten nachgewiesen hatten, sprachen wir Folgendes aus: „Es blieb also noch nachzuweisen, ob solche Isomerisation auch durch Einwirkung organischer Säuren und auch bei der Darstellung gemischter Aether aus den secundären Derivaten des Menthols und Carvomenthols entständen, und ob man überhaupt, und auf welche Weise secundäre Halogenverbindungen, die den secundären Alkohoen entsprechen, erhalten könnte."

Durch unsere weitere Untersuchung, ebenso auch durch die Untersuchungen anderer Forscher kann man jetzt die Frage über die Isolirung secundärer Derivate für genügend entschieden halten. Auf solch' eine Weise hat die Feststellung 
208 Kondakow: Synthesen unter Einw. Z Zinkchlorid etc.

eine Isomerisation durch uns, welche eine gewichtige Bedeutung für die richtige Feststellung der Constitution von Terpenkohlenwasserstoffen besitzt, wie wir glauben keinen geringen Dienst Wagner bei der Entscheidung der Constitution des Camphens und Wallach für die des Fenchens geleistet, wie es aus dem weiter Folgenden ersichtlich ist.

Bis zu unseren Untersuchungen, die sich auf die oben angeführte Isomerisation bezogen, hielten Bredt, Tiemann, Wagner, Semmler u. a. das Camphen für ein gleichartiges und echtes Derivat des secandären Borneols mit einer Doppelbindung zwischen den secundär-secundären Kohlenstoffatomen im Ringe.

Aus diesem Grunde wurde dem Camphen aus der $\mathrm{Zu}$ sammengehörigkeit mit dem Campher folgende Constitution zugeschrieben:<smiles>CC1CCCC1(C)C</smiles>

Bredt ${ }^{1}$ )<smiles>CC1C2C=CC(C2)C1(C)C</smiles>

Tiemann $\left.{ }^{2}\right)$

Ein Blick auf diese Constitutionsformeln des Camphens zeigt, wie sehr sich bei den Forschern die Ueberzeugung festgesetzt hat, dass die Derivate des Borneols sich nicht möglicherweise isomerisiren könnten, sondern direct in das Camphen umwandeln. Aber nicht genug, es wurde eine gleiche Constitution dem Camphen aus dem Borneol und dem Isoborneol zugeschrieben, nicht nur dann, als das letztere für ein Stereoisomeres ${ }^{3}$ ) oder auch ein chemisches Isomeres mit secundärem Oharakter des Borneols gehalten wurde, sondern auch dann, nachdem v. Baeyer zuerst im Isoborneol einen Körper mit tertiärem alkoholischen Charakter vermuthete und dieses endgültig von anderen Forschern als tertiärer Alkohol anerkannt wurde.

Die Anschauung über die Constitution des Camphens als ungesättigten Kohlenwasserstoffs mit secundär-secundären

1) Ber. 26, 3056 .

3) Das. 29, 544; 29, 697.

2) Das. 28, 2182. 
Kondakow: Synthesen unter Einw. v. Zinkchlorid etc. 209

Kohlenstoffatomen bei der Doppelbindung im Ringe war so allgemein angenommen, dass Wagner $\left.{ }^{1}\right)$, der sich eine lange Zeit mit der Oxydation dieses Kohlenwasserstoffs beschäftigte, nachdem er nicht im Stande war, alle sich dabei gebildeten Produkte zu erklären, dem Camphen eine neue Constitutionsformel gab:<smiles>CC(C)C(C)(C)C</smiles>

wiederum mit secundär-secundären Kohlenstoffatomen bei der Doppelbindung im Ringe, nebenbei gesagt einer so wenig gelungenen, dass er bald sich von ihr zu Gunsten der Formel von Bredt lossagte, wobei or in seinen Abhandlungen vollständig jede Gegenwart eines tertiären Kohlenstoffatoms bei der Doppelbindung im Ringe und eine Doppelbindung in der Seitenkette negirte. Allein schon nach einem Monate, als die letzte der erwähnten Abhandlungen in den Berl. Berichten abgedruckt war, giebt Wagner in dem Journal d. russ. chem. physik. Ges. 31, 680 dem Camphen eine andere Constitution, gerade der entgegengesetzt, welche er für richtig hielt, und spricht sich für die Gegenwart einer Doppelbindung in der Seitenkette mit einem tertiären Kohlenstoffatom aus, indem er seine Voraussetzung zum grössten Theil auf eine längst bekannte Thatsache stützt, dass, wenn das Camphen aus dem Isoborneol erhalten wird, es dem letzteren entsprechen muss.

$\mathrm{Zu}$ solch' einer Folgerung war nach den von uns veröffentlichten oben angeführten Untersuchungen nicht schwer zu gelangen, nachdem noch J ünger-Klages ${ }^{2}$ ) und Reychler ${ }^{3}$ ) nach uns constatirten, dass das Bornylchlorid, Chlorwasserstoffcamphen und Isobornylchlorid unter einander identisch sind Eine Thatsache, die noch früher von Riban festgestellt, von Kachler und Spitzer ${ }^{4}$ ) wiederholt und von $\operatorname{Arth}^{5}$ ) einer

1) Journ. d. russ. chem.-physik. Ges. 28, 64 (1896); 29, 124 (1897); Ber. 32, 2302 (1899).

2) Ber. 29, 544 (1896).

s) Ber. 29, 697; Bull. d. 1. g. chim. [3] 15, 366 (1896).

4) Anv. Chem. 197, 86; 200, 340 .

5) Ann. chim. [6] 7, 478.

Journal f. prakt. Chemie [2] Bd, 65. 
210 Kondakow: Synthesen unter Einw. v. Zinkchlorid etc.

näheren Ausführung unterzogen wurde. Das Verhalten des Isoborneols, welches sich im Borneol befindet, zu den wasserabspaltenden Agentien, wie $\left.\mathrm{P}_{2} \mathrm{O}_{5}, \mathrm{ZnCl}_{2}{ }^{1}\right), \mathrm{H}_{2} \mathrm{SO}_{4}$ ist längst aus den Untersuchungen von Wallach bekannt, und das des reinen Isoborneols aus den Untersuchungen von Bertram-Walbaum ${ }^{2}$ ), auf seine Beziehungen zur Bromwasserstoffsäure ist von Baeyer hingewiesen worden, welcher die Gelegenheit ergriff, sich für den tertiären Charakter des Isoborneols auszusprechen, was auch später von Wagner angenommen wurde; diese hatten nur eine untergeordnete Bedeutung und unterstützten nur die oben angeführten Annahmen.

Das sind nach unserer Auffassung die wesentlich wichtigen Thatsachen, die bei der Entscheidung über die Constitution des Camphens von Bedeutung waren.

Allein nicht so sieht diese Frage Wagner an, welcher behauptet, durch die Oxydation des Camphens mit Kaliumpermanganat zur richtigen Entscheidung über den Bau des Camphens gelangt zu sein.

Mir scheint es, dass nicht die Oxydation Wagner den Grund zur Erklärung des Baues von diesem Kohlenwasserstoff gab, sondern das Gesammtergebniss anderer, mehr überzengender, unbestreitbarer und gewichtigerer Thatsachen, unter anderen auch hauptsächlich meine Entdeckung, die er mit consequentem Schweigen übergeht, da die Oxydationsreaction mit dem Permanganat von Umlagerungen sowie Hydratation und Dehydratation ${ }^{3}$ ) begleitet ist und selbst nicht zu den Methoden gehört, durch welche man zur Bestimmung der Constitution ungesättigter Verbindungen gelangen würde; durch sie allein könnte man nicht zu richtigen Schlüssen kommen.

Die weiteren bestimmteren Angaben von Wagner über

1) Es muss bier constatirt werden, dass zuerst von mir die Beziehung des tertiären Amylalkohols zum Zinkchlorid beobachtet wurde. Compt. rend. d. l. soc. natur. a l'Univers. de Varsovie, 18. May 1891.

) Dies. Journ. [2] 49, 1 (1894).

3) Ich halte es nicht für überflüssig daran zu erinnern, dass Wagner früher kategorisch eine mögliche Umgruppirung und Hydratation bei der Oxydation mit dem Permanganat verneinte; jetzt lässt er sie schon zu und theilt auf diese Weise die Meinang anderer Forseher, unter anderen auch die unserige. (Dies. Journ. [2] 59, 287). 
Kondakow: Synthesen unter Einw. v. Zinkchlorid etc. 211

die Unterschiede der Camphene aus dem Borneol und Isoborneol und ihrer versehiedenen Constitution, wurden von ihm nach der Veröffentlichung neuerer Untersuchungen über das Fenchen, und nach der Gewinnung eines neven, dem secundären Borneol entsprechenden Camphen von Tschugaeff ${ }^{1}$ ) gemacht; im Grunde sind sie nur die Folge dieser Untersuchungen. Dieses verdient um so mehr die Aufmerksamkeit, als Wagner früher das Bornylen (unreines) in den Händen hatte, es aber nicht isoliren und untersuchen konnte, diese wichtige Thatsache übersah und sich nur unter Räthseln und Muthmaassungen bewegte. Was die Constitution anbelangt, welche jetzt Wagner dem Isoborneol und Isobornylen zuschreibt, so benöthigt dieselbe noch einer Bestätigung, da diese Verbindungen einen anderen Bau haben können, wenn auch nur einen solchen, wie er ihnen jetzt von Semmler ${ }^{2}$ ) gegeben wird, der das Isocamphen (Isobornylen) als ungesättigten Kohlenwasserstoff betrachtet, mit einem Pentamethylen- und Tetramethylenring, mit einer Doppelbindung in der Seitenkette.

Wenn sich Wagner's Constitutionsformel wirklich bestätigen sollte, so würde sie, um seine Worte zu gebrauchen, nur ,eine unwesentliche bezüglich der Lage der Aethylenbindung veränderte" Formel von Tiemann vorstellen. Welche von diesen beiden Constitutionsformeln sich als die richtige erweisen, oder ob man dem Isocamphen [Isobornylen] irgend eine andere Constitution geben soll, ist noch eine Frage der Zeit.

Aus allem oben Gesagten ist jedenfalls eins klar ersichtlich, dass die Camphene nicht zu den gesättigten Körpern gehören, wie $\mathrm{Marsh}^{3}$ ) annimmt, sondern zu den ungesättigten, wobei eines der Camphene die Doppelbindung im Ringe enthält und dem Borneol entspricht; das andere enthält die Doppelbindung in der Seitenkette, wie von uns in unserer Bemerkung ${ }^{4}$ ) nach dem Befunde der unten angeführten thatsächlichen Ergebnisse ausgesagt wurde, es entspricht dem Isoborneol.

1) Chem. Zeitg. 24, 519: Journ. d. russ. physik. u. chem. Ges, 32, 358 (1900); Ber. 33, 2124 (1900).

2) Ber. 33, 3420 (1900).

3) Chem. Centralbl. 1899, I, 790.

4) Chem. Zeitg. 25, Nr. 13, 1901. 
212 Kondakow: Synthesen unter Einw. v. Zinkehlorid ete.

\section{Ueber die Fenchene.}

Die Geschichte des Fenchens ist der des Camphens ungemein ähnlich. Wie dieses letztere für einen directen Abkömmling des Camphers und des Borneols gehalten wurde, so brachte es Wallach mit dem Fenchon in directe Beziehung und betrachtete es als ein Derivat des secundären Fenchylalkohols.

Wie man das Camphen bis zur letzten Zeit für structurgleich und nur in seinen optischen Eigenschaften für verschiedenartig hielt, ebenso unterscheiden sich nach Wallach die Fenchene nur nach ihrem Drehungsvermögen, wie es aus folgenden Citaten ersichtlich ist: „dass aus dem l-Fenchylalkohol zwei verschiedene Fenchene entstehen können, von denen das eine die hoch-, das andere die niedrigschmelzende Säure giebt" (Ann. Chem. 302, 373), von diesen entspricht die letztere nach Wallach dem optisch activen und die hochschmelzende Säure dem optisch inactiven (racemischen) Fenchen.

Dann weiter: „was die rechts- und linksdrehende Oxyfenchensäure anbelangt, so wird man keinen Augenblick zweifelhaft darüber sein, dass es sich bei diesen nicht um chemische, sondern um Raumisomerie handelt, denn Entstehung und Verhalten beider Säuren ist analog." (Ann. Chem. $302,385)$.

Die Untersucher nach Bredt, welcher dem Camphen zuerst eine Constitutionsformel gab, hielten es für einen Kohlenwasserstoff mit einer Doppelbindung im Ringe, ebenso schrieb Wallach dem Fenchen eine analoge Constitution zu. Es wurde oben darauf hingewiesen, dass Wagner ${ }^{1}$ ), welcher sich lange mit der Oxydation des Camphens befasste, bis zur letzten Zeit nicht die Gegenwart einer Doppelbindung in der Seitenkette desselben anerkennen wollte, wie es aus seinen Worten folgt: „Wenn wir alles oben ausgeführte zusammenhalten (d. h. die Oxydation des Camphens), so muss man anerkennen, dass die von uns erhaltenen Thatsachen, wenn sie auch nicht endgültig alle Zweifel beseitigen, so doch die Folgerung einer Uneinheitlichkeit des Camphens wenig wahrscheinlich machen; im Gegentheil, die Oxydationsprodukte sind derartig, dass man sie

1) Journ. russ. phys. chem. Ges. 28, 78. 
Kondakow: Synthesen unter Einw.v. Zinkchlorid etc. 213

für aus einem und demselben Kohlenwasserstoff entstanden halten muss:<smiles>[14CH3]C1[CH][SiH2]1</smiles>

Noch später spricht Wallach ${ }^{1}$, als er die Oxydationsprodukte des Fenchens, im Besondern die entstandene Oxyfenchensäure prüft, Folgendes aus: „Eine solche würde sich durchsichtiger aus einem Fenchen ableiten lassen, dem dieGruppirung $\mathrm{C}_{8} \mathrm{H}_{14}: \mathrm{C}: \mathrm{CH}_{2}$ zukäme. Falls Fenchen ohne Umlagerung aus Fenchylchlorid entsteht (und eine solche anzunehmen, ist einstweilen keine Veranlassung), würde dann aber für den Fenchylalkohol lediglich die Formel $\mathrm{C}_{8} \mathrm{H}_{14}: \mathrm{C}(\mathrm{OH}) \mathrm{CH}_{3}$ oder $\mathrm{C}_{8} \mathrm{H}_{14}$ : $\mathrm{CHCH}_{2}(\mathrm{OH})$ möglich sein, die beide völlig ausgeschlossen sind, da ja der Fenchylalkohol ein secundärer Alkohol ist"... Weiter: „Nimmt man nun, wie es oben geschehen ist, für das Fenchen die Formel<smiles>CC1CC1[14CH3]</smiles>

an, so müsste die Oxydation bei dem $\mathrm{CH}_{3}$ unter gleichzeitiger Sprengung der Doppelbindung und Wasseraufnahme einsetzen. In diesem Falle müsste die $\alpha$-Oxysäure

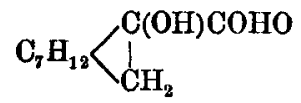

entstehen, bei deren weiterer Oxydation sich das Keton

bilden würde."<smiles></smiles>

"Wie steht es aber mit dem Fenchen?" fragt sich W allach weiter ${ }^{2}$ ) und antwortet darauf: „Ich habe neulich ${ }^{3}$ ) für Fenchen die Formel<smiles>CC(C)(C)C1CCC2CCCC21</smiles>

1) Ann. Chem. 300, 320 (1898).

2) Das. 302, $385 . \quad$ s) Das. 300, 320. 
214 Kondakow: Synthesen unter Einw. v. Zinkchlorid etc.

den Betrachtungen zu Grunde gelegt. Das Symbol weisst zwei asymmetrische Kohlenstoffatome auf, das Fenchen könnte also in zwei Modificationen der beobachteten Art bestehen;" Nur das ungleiche Verbalten des rechts- und links- Fenchen gegen das Kaliumpermagannat spricht nach Wallach ,gegen physikalische und für chemische Isomerie", als ob es darauf hinwies, dass diese Fenchene sich unter einander wie gesättigte und ungesättigte Verbindungen verhalten. Dieses Alles zusammenfassend, sagt Wallach: „Man ist demnach auf Grund des bisherigen Beobachtungsmaterials genöthigt anzunehmen. dass $d$ - und l-Fenchen auch nur physikalisch verschiedene Modificationen eines Kohlenwasserstoffs mit einer Aethylenbindung sind. Daraus würde dann aber weiter die wichtige Thatsache zu entnehmen sein, dass Aethylenbindungen bei stereoisomeren Substanzen unter dem Einfluss vom Permanganat mit ganz verschiedener Leichtigkeit gelöst werden können und damit würde tür die bekannte Anwendung von Permanganat als diagonostischem Mittel für den Nachweis ron Aethylenbindungen eine beachtenswerthe Einschränkung nothwendig werden."

Schimmel in seinem Bericht (1900, October-Heft, 74), unsere Arbeit referirend, giebt unrichtiger Weise an, dass Wallach vor uns im Fenchen zwei chemische Isomere anerkannt hat. Soviel uns bekannt, und aus den angeführten Citaten ersichtlich ist, erkennt $W$ allach das Fenchen nur als ein Gemisch zweier Stereoisomere aber nicht chemischer Isomere an. Erst nach unseren Untersuchungen war Wallach gezwungen, im Fenchen ein Gemisch ron zwei chemischen Isomeren anzuerkennen.

Wie das Bornylchlorid bis zu meinen Untersuchungen, ungeachtet der in der Litteratur enthaltenen widersprechenden Angaben, für ein secundäres Chloranhydrid des eigentlichen Borneols gehalten wurde, eben so wurden das Fenchylchlorid und sogar das Chlorwasserstofffenchen von Wallach für secundäre Chloranhydride angesehen.

In solcher Lage befand sich die Frage über die Constitution des Fenchens am Ende des Jahres 1898, d. h. zu der

1) Ann. Chem. 302, 385--387. 
Kondakow: Synthesen unter Einw. v. Zinkehlorid etc. 215

Zeit, als von uns die Isomerisation der secundären hydroaromatischen Alkohole Menthomenthol und Carvomenthol in tertiäre festgestellt wurde und dabei die Behauptung ausgesprochen wurde, dass eine gleiche Umlagerung auch bei den anderen secundären Alkoholen zu erwarten sei. „Nach dieser Regel ist zu erwarten, dass der Fenchylalkohol ${ }^{1}$ ), wenn die Formel

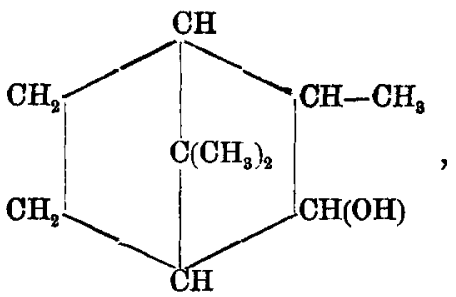

welche Wallach ihm zuschreibt, richtig ist, sich ähnlich dem Menthol und Carvomenthol isomerisiren muss.

Daher müssen das Fenchylchlorid und das Chlorwasserstofffenchen, welche von $W$ allach erhalten wurden, ebenso auch ihre anderen Halogenderivate identisch sein und dem tertiären Fenchylalkohol entsprechen.“")

Nach uns sprachen Bertram und Helle in ihrer Abhandlung, "Ueber den Isofenchylalkohol ${ }^{(3)}$ ), nachdem sie die Eigenschaften des erhaltenen Fenchens beschrieben hatten, Folgendes aus „Ganz geklärt sind hier die Verhältnisse noch nicht und es bleibt festzustellen, ob, wie Wallach ${ }^{4}$ ) annimmt, der Uebergang rom Fenchylalkohol über das Fenchylchlorid in Fenchen ohne Umlagerung erfolgt, oder ob bei der Einwirkung des Phosphorpentachlorids auf den Fenchylalkohol, wie Kondakow und Lutschinin vermuthen, Isomeration eintritt, so dass sowohl das Fenchylchlorid, als auch das daraus dargestellte Fenchen dem angewandten Fenchylalkohol nicht mehr entsprechen."

Kaum zwei Monate nach dem Erscheinen der erwähnten Abhandlung von Bertram und Helle erschien unsere Unter-

1) „Dieser Alkohol kann beim Auseinandertreten der Diagonalbindung Derivate geben, die dem Ortho- und Metacymol entsprechen.“

2) Dies. Journ. [2] 60, 279 (1899).

3) Das. 61, 299 (1900).

4) Ann. Chem. 302, 320. 
216 Kondakow: Synthesen unter Einw. v. Zinkchlorid etc.

suchung über die Derivate des Fenchylalkohols, in welcher wir nicht nur den Beweis bis in die kleinste Einzelheit für die Bestätigung unserer früher ausgesprochenen Behauptung gaben, sondern die Constitution des Fenchens feststellten.

So bewiesen wir, dass die Haloïdanhydride des Fenchylalkohols zum grössten Theil aus Haloidanhydriden des tertiären Fenchylalkohols (Isofenchylalkohol) bestehen und identisch sind mit den Haloidwasserstoffverbindungen des Fenchens mit einer nur sehr geringen Beimengung von Haloidanhydriden, die dem secundären Alkohol entsprechen. Weiter wurde von uns bewiesen, dass das Fenchen wenigstens aus zwei chemischen Isomeren besteht, nicht aber aus geometrischen, wie Wallach annahm; das eine von denselben wurde von uns etwas später rein isolirt und beschrieben ${ }^{1}$ ). Als auf solche Weise die Frage über die Zusammensetzung der Haloidanhydride des Fenchylalkohols und über die Constitution des Fenchens unwidersprechlich entschieden war, erschien die 51. Abhandlung von $W_{\text {allach}}{ }^{2}$ im Druck, über deren Einlieferung in die Redaction der Annalen und darüber, wann sie von dem Verfasser geschrieben, keinerlei Angaben gemacht sind. Es ist jedoch nicht schwer festzustellen, dass in dieser Abhandlung, welche im Februar 1901 erschien, also ein halbes Jahr nach unseren Untersuchungen, genau dasselbe behandelt wird, sowohl über das Fenchylchlorid als auch das Fenchen, wie in unserer Veröffentlichung, ohne jeden Hinweis auf unsere Arbeit, als ob diese gar nicht vorhanden sei.

Zur Feststellung dessen, in wiefern die Schlüsse von Wallach über das Fenchylchlorid und Fenchen wirklich mit den unseren identisch sind, will ich folgende Stelle aus unserer Abhandlung ausschreiben:

„Aus dem Fenchylalkohol werden in der That zwei Chloride erhalten, ein secundäres und ein tertiäres; letzteres kann wiederum seinerseits einen zweifachen Bau haben. Mit Annahme der von Wallach für den Fenchylalkohol ange-

1) Chem. Zeitg. 25, Nr. 13 (1901).

2) Ann. Chem. 315, 373 (1901). 
Kondakow: Synthesen unter Einw. v. Zinkchlorid etc. 217 gebenen Formel wird das entsprechende Chlorid folgende Constitution besitzen:<smiles>CC1C2CC3CC1C(Cl)C3C2</smiles>

Das oben angeführte feste Fenchylchlorid wird wahrscheinlich auch einen solchen Bau haben.

Bei der Isomerisation des secundären Chlorides können, durch das Fenchen gehend, aus ihm die zwei folgenden tertiären Chloride erbalten werden:
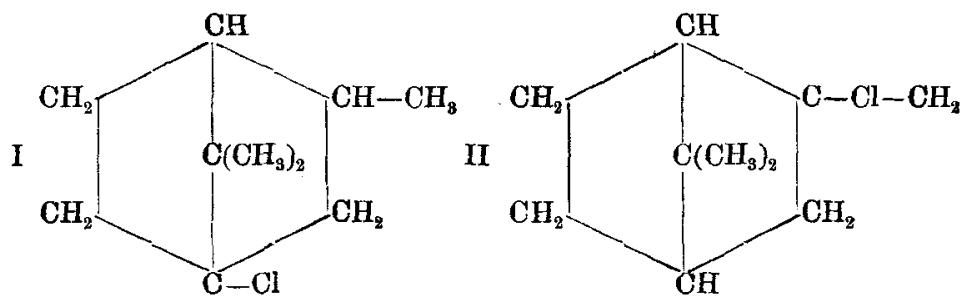

Das letztere kann noch ein drittes tertiäres Chlorid geben:

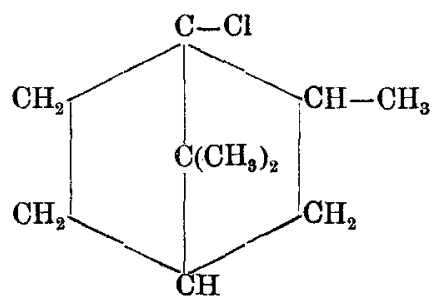

Das sogenannte Fénchylchlorid kann daher ein Gemisch aller vier möglichen Chloride darstellen oder ein Gemisch des secundären mit drei tertiären sein, oder nur aus einem der tertiären bestehen. Mehr Wahrscheinlichkeit hat die Voraussetzung, dass das Fenchylchlorid aus einem Gemisch des secundären mit einem tertiären der Formel II besteht. Aus solchem Chloridgemisch kann beim Bearbeiten mit Laugen bei mässiger Temperatur der Chlorwasserstoff sich vornehm- 
218 Kondakow: Synthesen unter Einw. v. Zinkchlorid etc.

lich vom tertiären Chlorid $^{1}$ ) abspalten und mehrere isomere Fenchene geben.

So kann das tertiäre Fenchylchlorid der Constitution II folgende drei Fenchene liefern:
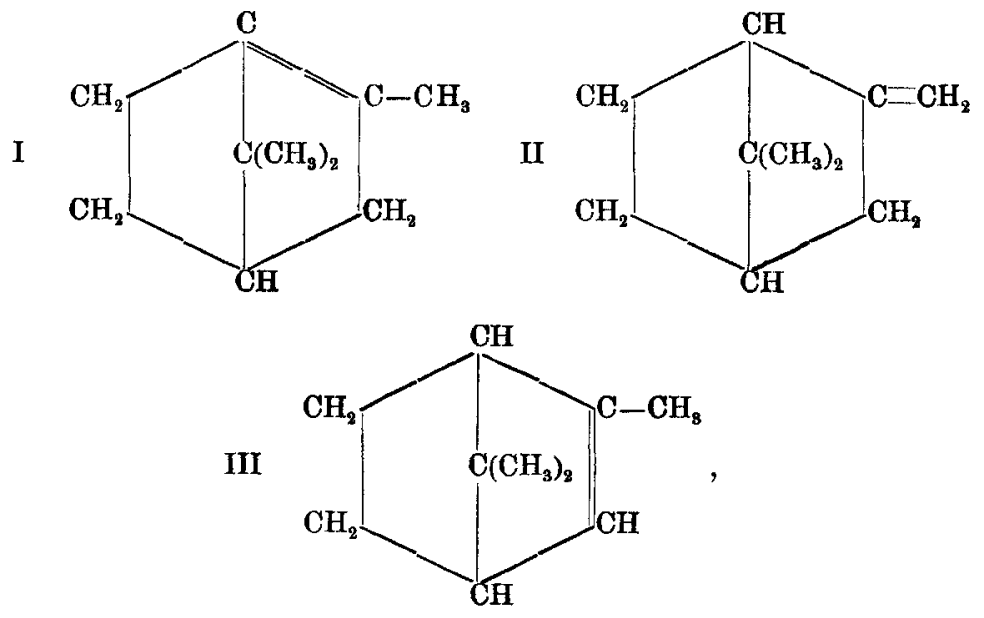

von denen das dritte sich auch beim Abspalten von Chlorwasserstoff aus dem secundären Chlorid bilden kann, wie es Wallach annimmt.

Ausser diesen drei Fenchenen kann aus dem secundären Chlorid noch ein viertes Fenchen gebildet werden:

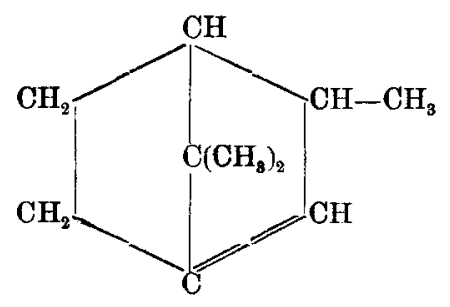

Diese vier Fenchene können am wahrscheinlichsten beim Abspalten von Chlorwasserstoff aus dem sogenannten Fenchylchlorid erwartet werden. . . . ."

1) Dieser Anschauung sind auch andere Forscher, nur bedarf sie einer eingehenden Controlle, da diese Regelmässigkeit nicht durch alle bekannten Thatsachen bestätigt wird. 
Kondakow: Synthesen unter Einw. v. Zinkchlorid etc. 219

Ganz dasselbe sagt jetzt Wallach, welcher sich ganz von Allem dem lossagt, was er kategorisch früher über das Fenchylchlorid und Fenchen behauptet hat. Ehedem hielt Wallach das Fenchen für ein Gemenge aus geometrischen Isomeren, jetzt aber erscheint es ihm als Gemisch von drei chemischen Isomeren: „Denn nach den neuern Beobachtungen ist es viel wahrscheinlicher geworden, dass die verschiedene Leichtigkeit, mit der Fenchene sich oxydiren lassen, nicht auf die stereoisomeren, sondern auf die chemisch isomeren Formen zurückzuführen ist, die sich in verschiedenen Fenchenpräparaten vorfinden können." ${ }^{1}$ ) Vorher hielt Wallach das Fenchylchlorid für ein chemisch einheitliches secundäres Chlorid, jetzt aber bezeichnet er es als "ein Gemisch verschiedener Chloride".... Weiter fügte er hinzu: „Diesen verschiedenen Chloriden correspondiren augenscheinlich verschiedene Fenchene, aber es ist natürlich daneben nicht ausgeschlossen, dass aus ein und demselben Fenchylchlorid durch Salzsäureabspaltung zwei structuris omere Fenchene sich bilden können." „Fenchylchloride, welche sie unter verschiedenen Bedingungen bilden, können bald dem bei $45^{\circ}$ schmelzendem gewöhnlicbem Fenchylalkohol, bald dem bei $61^{\circ}$ schmelzenden Isofenchylalkohol entsprechen und die daraus gewonnenen Fenchene werden entsprechende Isomerien aufweisen.“2)

Bis zu unserer Abhandlung negirte Wallach, wie oben angeführt, die Möglichkeit einer Gegenwart von Isomeren im Fenchen mit einer Doppelbindung in der Seitenkette, jetzt aber spricht er sich folgendermassen aus: „Früher habe ich die Annahme der Bildung eines solchen Kohlenwasserstoffs mit semicylischer Methylenbindung da, wo die Entstehung einer Doppelbindung innerhalb des Kohlwasserstoffringes möglich erschien, als wenig wahrscheinlich geglaubt verwetten zu müssen. Nachdem ich aber inzwischen bei anderer Gelegenheit den experimentellen (?) Beweis dafür habe beibringen können, dass die Entstehung gerade dieser Bindung, auch wenn andere Möglichkeiten vorhanden sind, unter Umständen sogar bevorzugt wird, liegt nicht nur kein Grund mehr vor . . . ."

1) Amu. Chem. 315, 301.

2) Das. 315, 280-232. 
220 Kondakow: Synthesen unter Einw. v. Zinkchlorid etc.

Bezüglich der Entstehung dieses Fenchens aus D-l-Fenchylalkohol spricht Wallach weiter: „Das Zustandekommen des Kohlenwasserstoffs III aus dem D-1-Fenchylalkohol ist dann natürlich nur zu erklärlich, dass der zuerst entstehende Kohlenwasserstoff I oder II Bindungsverschiebung erleidet. Das Auftreten deratiger Bindungsverschiebungen bei den Terpenkohlenwasserstoffen und ihren Derivaten habe ich ja aber im Beginne meiner Arbeiten gerade als charakteristisch für die ganze Gruppe nachgewiesen. Es hat nichts Auffallendes, wenn aus dem aus dem Fenchylalkohol zuerst auftretenden normalen Chlorid unter Abspaltung und Wiederaufnahme von Chlorwasserstoff successive verschiedene Fenchylchloride und dann auch Fenchene entstehen würden.“" 1 )

Nach diesem Citat scheint es, als ob W allach, nicht wir, diese Isomerisation festgestellt hätte und als ob er sie schon längst in seinen Arbeiten vorausgesehen hätte. Diese seine Behauptung wird erstens dadurch widerlegt, dass nicht in einer seiner $\mathrm{zu}$ verschiedenen Zeiten erschienenen Abhandlungen bis zur Druckerscheinung unserer Entdeckung die Erwähnung einer Voraussehung von Isomerisation gemacht wird, zweitens dadurch, dass, wenn Wallach sie wirklich im gegebenen Fall vorausgesehen hätte, kein Grund vorlag, seine $\nabla$ oraussehung nicht früher zur Erklärung der Constitution des Fenchens zu benutzen; warum hatte er es dann nöthig Hypothesen über den Mechanismus bei der Oxydation dieses Kohlenwasserstoffs zu bauen, ebenso auch die Fenchene nur nach ihren optischen Eigenschaften zu unterscheiden, wobei er sogar ganz die Möglichkeit von chemischen Isomeren in diesem Kohlenwasserstoff negirte - alles dieses bis zum Erscheinen unserer Abhandlung?

Zur Frage über die Constitution des Fenchens zurückkehrend, ist es nothwendig daran ' $z u$ erinnern, dass die Frage zuerst von uns entschieden worden ist, wie man aus dem obenangeführten Citat unserer Abhandlung ${ }^{2}$ ) ersehen kann, ebenso auch aus der in der Chemiker Zeitung ${ }^{3}$ ) veröffentlichten Bemerkung.

I) Ann. Chem. 315, 300.

2) Dies. Journ. [2] 62, 14.

3) Chem. Zeitung 1901, Nr. 13. 
Kondakow: Synthesen unter Einw. v. Zinkchlorid etc. 221

Wallach sagt jetzt zu dieser Frage folgendes: „Ich will zunächst über die muthmassliche Constitution der Fenchene aus d-l-Fenchlyalkohol sprechen. Der Uebergang des Alkohols in Fenchen kann im ersten Schritt auf zweierlei Weise erfolgen:

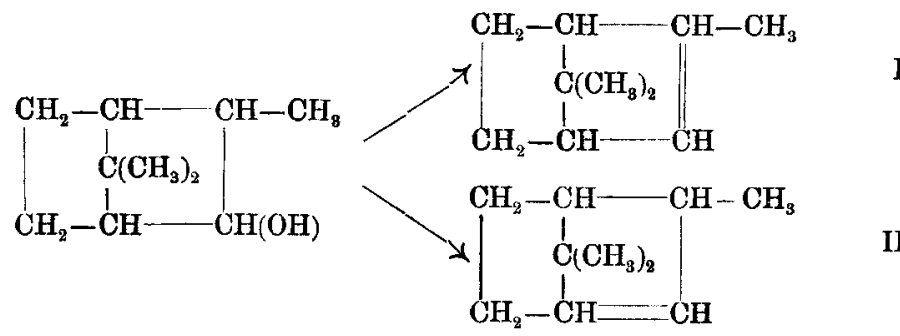

Darauf Bildung der Oxysäure vom Schmelzp. $152^{\circ}$ und von der Constitution<smiles>O=C(O)C1(O)CC2CCC1C2</smiles>

Jetzt erklärt Wallach sie so: „Auch habe ich damals schon bemerkt (Ann. Chem. 300, 321), dass man diese Formel allenfalls mit der oben mit I bezeichneten für Fenchen vereinigen kann, falls man für die Entstehung der Säure eine Erklärung giebt, wie Baeyer sie für die Bildung von Nopinsäure aus Pinen benutzt. Aber diese Erklärung hat doch etwas Gezwungenes. Viel besser würde sich die Bildung der Oxysäure aus einem Kohlenwasserstoff<smiles>C=C1CC2CCC1C2(C)C</smiles>

ableiten lassen, wie ich loc. cit. (S. 320 $)^{1}$ ) schon hervorgehoben habe." ${ }^{2}$ )

Ausser diesen drei Fenchenen sind nach Wallach aus dem Fenchylalkohol noch folgende vier Fenchene möglich:

1) In diesem Citat wird gerade das Gegentheil behauptet.

2) Ann. Chem. 315, 299. 
222 Kondakow: Synthesen unter Einw. v. Zinkchlorid etc.<smiles>CC1=C2CCC(C2)C1C</smiles>

IV<smiles>[R2]C1CCC(C)C1C</smiles>

VI<smiles>[R17]C1CCC=C1C(C)C</smiles>

$\mathrm{V}$<smiles>CC1CC2CCC1C2</smiles>

VII

Diese Zulassung von $\mathrm{W}$ allach ist eine stillschweigende Anerkennung der von uns festgestellten Isomerisation der Derivate des Fenchylalkohols und ebenso auch der Constitution, wie sie von uns dem Fenchylchlorid gegeben wurde.

Zum Schluss gelangt Wallach zur Annahme, dass ,im Rohfenchen mindestens drei Fenchene enthalten sein können, nämlich zwei schwer oxydirbare und eine leicht oxydirbare Form." "1) Diese werden von ihm mit den Zahlen I, II, III bezeichnete $d . h$. gerade die drei Fenchene, von den vier Isomeren, deren wahrscheinliche Gegenwart wir im Rohfenchen annahmen.

Eine weitere Nebeneinanderstellung unserer Abhandlungen mit denen von Wallach will ich nicht machen, da aus dem Obenangeführten genügend klar ersichtlich ist, wem von uns die Priorität in Betreff der Constitution sowohl des Fenchens als auch des Fenchylchlorids zukommt.

Alles über das Camphen und Fenchen Obenangeführte führt zu dem Gedanken, dass zur Aufklärung ihrer Constitution eine wesentliche Bedeutung der von uns geführte Nachweis der Isomerisation secundärer hydroaromatischer Alkohole zu tertiären hatte, da von diesem Augenblicke an es möglich war, mit einer bestimmten Gewissheit die Entstehung dieser Kohlenwasserstoffe aus den Derivaten tertiärer Alkohole festzustellen, folglich auch zu vermuthen, dass im Rohcamphen und RohFenchen Isomere enthalten sein können, mit der Doppelbindung in der Seitenkette.

1) Ann. Chem. 315, 284. 
Kondakow: Synthesen unter Einw. v. Zinkchlorid etc. 223

Dieses nöthigt, seinerseits vom neuen durchzusehen, wie sich diese neuen Thatsachen zu dem Ergebnisse über die Oxydation dieser Kohlenwasserstoffe verhalten, dass die Oxydationsmethode mit dem Permanganat allein, wie oben gezeigt wurde, nicht zu richtigen Schlüssen führen konnte und bis zur Jetztzeit nicht geführt hat.

Die weiter unten beschriebenen Versuche, welche bedingt wurden, als das Camphen und Fenchen noch für Kohlenwasserstoffe mit einer Doppelbindung im Ringe gehalten wurden und im Zusammenhang mit den Versuchen über Tetrahydro-Toluol, Menthene, Dipenten, Pinen und andere ausgeführt wurden, bestätigen auf das Beste, dass das käufliche Camphen (Isocamphen resp. Isobornylen), welches für einen Kohlenwasserstoff mit der Doppelbindung in dem Ringe zwischen den secundär-secundären Kohlenstoffatomen gehalten wurde, fast ausschliesslich aus Isomeren mit einer Doppelbindung in der Seitenkette besteht; das Fenchen aus dem Fenchylchlorid und aus anderen Haloidanhydriden des Fenchylalkohols, welches von uns untersucht wurde, besteht aus zweien Isomeren, von denen das eine die Doppelbindung im Ringe, das andere in der Seitenkette besitzt.

\section{Experimenteller Theil.}

I.

Synthese von Estern des tertiären Bornylalkohols (Isobornylalkohol) aus Camphen mit organischen Säuren.

(Gemeinschaftlich mit Herrn E. Lutschinin.)

Bei der Synthese der weiter unten beschriebenen Ester benutzten wir die Methode von Kondakow ${ }^{1}$ ), die er für die Darstellung des Ester der tertiären Alkohole aus der Olefinreihe vorschlug. So wie damals, so wurden auch jetzt der Kohlenwasserstoff Isocamphen und die Säure in Molekular-

1) „Ueber die Synthese in der Fettreihe unter der Einwirkung von Zinkchlorid." Warschau 1894. Bull. soc. chim. [3] 7, 576 (1892). 
224 Kondakow: Synthesen unter Einw. v. Zinkchlorid etc.

mengen genommen, wenn die Säure sich wenig in Wasser löste, das $1 \frac{1}{2}$ - sogar das 2 fache Molekulargewicht der Säure, wenn sie sich gut durch Wasser entfernen liess. Das Zinkchlorid wurde, wie auch bei den früheren Versuchen, zu 2 Grm. auf jede $10 \mathrm{Grm}$. Kohlenwasserstoff genommen. Zur Gewinnung der Ester aus Säuren mit kleinem Molekulargewicht war kein Erwärmen nöthig, da das Gemisch von Säure und Camphen beim Eintragen des Zinkchlorids sich von selbst erwärmte, bei den höheren Säuren wurde das Gemisch zur Beschleunigung der Reaction bis auf $50^{\circ}$ erwärmt. Die Reaction endet beim Einhalten dieses Verfahrens in wenigen Minuten und giebt an Ester die theoretisch berechnete Menge. Ganz gleiche Resultate kann man erzielen, wenn man das Gemisch zur Esterification bei gewöhnlicher Temperatur auf kürzere oder längere Zeit stehen lässt, jedentalls nicht länger als 24 Stunden.

Zur Reinigung der erhaltenen Ester vom Zinkchlorid und den Säuren wird mit Wasser gewaschen und unter vermindertem Druck destillirt. Bei der Destillation der Ester aus dem Camphen wurde in allen unten beschriebenen Fällen in den ersten Antheilen des Destillats immer eine geringe Menge von Camphen erhalten, welches nicht in Reaction getreten war und sich während der Destillation verflüchtigte. Diese ist wahrscheinlich Tschugaeff's-Camphen, welches in dem käuflichen Camphen enthalten ist. Das Camphen, welches uns für die Versuche diente, wurde von Schimmel verschrieben, es siedete bei $158^{\circ}-159^{\circ}$ und schmolz zwischen $49^{\circ}-50^{\circ}$. Seine anderen Eigenschaften, wie Brechungsvermögen und Drehungsvermögen wurden auch bestimmt, sind aber leider verloren gegangen, so dass die Schlüsse über die optischen Eigenschaften der Ester nicht vollständig und genau sein können und noch zur Vervollständigung der Versuche bedürfen.

Ameisensaurer Isobornylester.

Eine farblose klare Flüssigkeit von angenehmem Aethergeruch, welcher an Baldrian erinnert.

Unter $19 \mathrm{Mm}$. Druck siedete er bei $106^{\circ}$.

Die Bestimmung des spec. Gew. ergab: 
Kondakow: Synthesen unter Einw. v. Zinkchlorid etc. 225

$$
\text { d. } \frac{20^{\circ}}{20^{\circ}}=1,0127 . \quad \quad d \frac{20^{\circ}}{4^{0}}=1,010 .
$$

Das Brechungsvermögen war:

$$
n_{D}=1,47164 \text {. }
$$

Molekularrefraction.

$$
\begin{array}{cc}
\text { Berechnet für } \mathrm{C}_{10} \mathrm{H}_{18}(\mathrm{COHOH}): & \text { Grefunden: } \\
\text { Nach Courady's Zahlen } & \mathbf{5 0 , 2 8}\left(20^{\circ}\right) \\
50,39 & 50,41\left(\frac{20^{\circ}}{4^{\circ}}\right) .
\end{array}
$$

Er ist optisch inaktiv.

Der Vergleich unseres Esters mit dem Isobornylameisensäureester von Bertram und $\mathrm{Walbaum}^{1}$ ) zeigt ihre völlige Aehnlichkeit, und es sind nur sehr geringe Unterschiede mit dem Bornylester von Bruylants ${ }^{2}$ ), Lafont ${ }^{3}$ ), Oliviero ${ }^{4}$ ) zu bemerken.

\section{Essigsäureisobornylester.}

Ist eine farblose klare Flüssigkeit mit einem Baldriangeruch; siedet unter $12 \mathrm{Mm}$. bei $102^{\circ}$ und ist optisch inaktiv.

Das spec. Gew. beträgt:

$$
d \frac{20^{\circ}}{20^{\circ}}=0,9858 . \quad d \frac{20^{\circ}}{4^{0}}=0,9841 .
$$

Die Bestimmung des Brechungsvermögens ergab:

$$
n_{D}=1,46494 \text {. }
$$

Molekularrefraction.

Berechnet für $\mathrm{C}_{10} \mathrm{H}_{16} \mathrm{COHOCH}_{3}$ :

Gefunden:

Nach Conrady's Zahlen $55,0 \quad 54,94\left(20^{\circ}\right) ; 55,08\left(\frac{20^{0}}{4^{0}}\right)$.

Seinen Eigenschaften nach ist auch dieser Ester dem Isobornylessigsäureester von Bertram und Walbaum ähnlich, von dem Bornylessigsäureester von $\mathrm{M}_{\text {ontgolfier }}{ }^{5}$ ), Kahler und Spitzer ${ }^{6}$ ), Bertram und Wahlbaum ${ }^{7}$ ), Bouchardat-

1) Dies. Journ. [2] 49, 7 (1894).

2) Ber. 11, 456.

8) Ann. chim. [6] 15, 167.

$\left.{ }^{4}\right)$ Compt. rend. 117, 1026.

3) Ann. chim. [5] 14, 50.

7) Ber. 26, [2] 685.

Journal f. prakt, Chemie [2] Bd, 165.

8) Ann. Chem, 200, 352. 
226 Kondakow: Synthesen unter Einw. v. Zinkchlorid etc. Lafont ${ }^{1}$ ) unterscheidet er sich nur dadurch, dass er nicht krystallisirt.

Isobornylisobuttersäureester.

Dieser Ester stellt eine farblose, recht dickfüssige Substanz vor, mit dem Geruch nach der Baldrianwurzel, er siedet bei $19 \mathrm{Mm}$. zwischen $132^{\circ}-133^{\circ}$.

Las spec. Gew. betrug:

$$
\text { d } \frac{20^{\circ}}{20^{\circ}}=0,9628 . \quad \quad \text { a } \frac{20^{\circ}}{4^{0}}=0,9611 .
$$

Das Brechungsvermögen betrug:

$$
n_{D}=1,46276 \text {. }
$$

Molekularrefraction.

$$
\text { Berechnet für } \mathrm{C}_{10} \mathrm{H}_{18} \mathrm{C}_{4} \mathrm{H}_{8} \mathrm{O}_{2} \text { : }
$$

Gefunden:

Nach Conrady's Zahlen $64,20 \quad 64,02\left(20^{0}\right) ; 64,14\left(\frac{20^{0}}{4^{0}}\right)$.

Er ist optisch inactiv.

Isovaleriansaurer Isobornylester.

Dieser Ester stellt eine dicke farblose Flüssigkeit mit ausgesprochenem Baldrianölgeruch vor. Bei der. Darstellung dieses Esters wurde anfangs eine grünliche Flüssigkeit erhalten, die nach dem $A b$ waschen des Zinkchlorids farblos wurde. Der Ester siedet bei $13 \mathrm{Mm}$. bei $132^{\circ}-133^{\circ}$ und wirkt auf Katzen wie das ätherische Baldrianöl, was zur Annahme berechtigt, dass man es an Stelle dieses Präparates in der Medicin gebrauchen kann und sogar mit grösserer Bequemlichkeit.

Die Isovaleriansäure, welche zu dieser Synthese genommen wurde, hatte folgende Eigenschaften:

$$
{ }^{\alpha}{ }_{D}=+2^{\circ} . \quad[x]_{D^{20}}=+2^{0} 8^{\prime} .
$$

Das spec. Gew. des Esters war:

$$
d \frac{20^{\circ}}{20^{\circ}}=0,9523 . \quad d \frac{20^{0}}{4^{0}}=0,9506 .
$$

Die Bestimmung des Brechungsvermögens ergab:

$$
n_{D}=1,46038 \text {. }
$$

\section{Molekularrefraction.}

Berechnet für $\mathrm{C}_{10} \mathrm{H}_{16} \mathrm{C}_{5} \mathrm{H}_{10} \mathrm{O}_{2}$ :

Nach Conrady's Zahlen $68,81 \quad 68,52\left(20^{\circ}\right) ; 68,63\left(\frac{20^{0}}{4^{0}}\right)$.

') Ann. chim. [6] 9, 509 . 
Kondakow: Synthesen unter Einw. v. Zinkchlorid etc. 227

Die Restimmung des Drehungsvermögens gab:

$$
\begin{array}{ll} 
& \alpha_{D}=+45^{\prime} \\
{[\alpha]_{D\left(20^{\circ}\right)}=+47^{\prime} .} & {[\alpha]_{\frac{20^{0}}{4^{0}}}=+47^{\prime} .}
\end{array}
$$

Alle oben beschriebenen Ester gaben bei der Verseifung einen Isobornylalkohol mit dem Schmelzpunkt von $212^{\circ}$ in eingeschmolzenem Röhrchen, er sublimirt sehr leicht in Form federartiger Krystalle.

Die Bestimmung des Drehungsvermögens dieses Alkohols, erhalten nach der Verseifung des Ameisensäureesters, ergab folgendes:

$$
\begin{gathered}
s=0,3738 ; \quad L=12,7395 ; \quad p=2,85 \% ; \quad d=0,7964 ; \quad t=20^{\circ} ; \\
\quad l=2 \text { Dcm. } \\
\alpha_{D}=-10^{\prime} . \quad[\alpha]_{D}=-7^{\circ} 20^{\prime} .
\end{gathered}
$$

II.

Synthese von Estern des tertiären Fenchylalkohols (Isofenchylalkohol) aus Fenchen mit organischen

\section{Säuren.}

Von den Ester des Isofenchylalkohols sind bis jetzt nur der Essigsäureester von Bertram und Helle $^{1}$ ) dargestellt worden, entweder nach dem patentirten Verfahren von Bertram aus dem Fenchen oder aus dem Isofenchylalkohol selbst.

Für die Darstellung der weiter zu beschreibenden Ester des Isofenchylalkohols gebrauchten wir das Verfahren von Kondakow. Das Fenchen, welches uns zu diesem Zwecke diente, war verschiedener Herkunft mit verschiedenem Drehungsvermögen. Am meisten gebrauchten wir das Fen. chen aus dem Fenchylbromid. Bei der Bereitung der untenbeschriebenen Ester des Isofenchylalkohols aus dem Fenchen nach unserem Verfahren hinterblieb immer ein Theil des Fenchens, welches nicht in Reaction getreten war und bei der fractionirten Destillation leicht vom Ester getrennt werden kann. Der nach der Reaction nachgebliebene Kohlenwasserstoff wurde von uns in reinem Zustande abgeschieden und beschrieben. ${ }^{2}$ )

1) Dies. Journ. [2] 61, 302 (1900).

2) Chem. Zeitung 1901, 25, Nr. 13. 
228 Kondakow: Synthesen unter Einw. v. Zinkchlorid ete.

Isofench ylameisensäureester.

Dieser Ester ist farblos und klar, er hat einen Geruch, welcher an Baldrian erinnert, und siedet bei $17 \mathrm{Mm}$. bei $98^{\circ}$.

Das spec. Gewicht betrug:

$$
d \frac{20^{0}}{20^{0}}=0,9847 . \quad d \frac{20^{0}}{4^{0}}=0,9829 .
$$

Die Bestimmung des Brechungsvermögens ergab:

$$
n_{D} 1,45979 \text {. }
$$

Molekularrefraction.

Berechnet für $\mathrm{C}_{10} \mathrm{H}_{10} \mathrm{COHOH}$ :

Gefunden:

Nach Conrady's Zahlen $50,39 \quad 50,59\left(20^{\circ}\right) ; 50,68\left(\frac{20^{\circ}}{4^{\circ}}\right)$.

Die Bestimmung des Drehungsvermögens ergab:

$$
\begin{array}{cl}
{[\alpha]_{D^{200}}=-32^{\circ} 1^{\prime} .} & \alpha_{D}=-31^{\circ} 29^{\prime} . \\
{[\alpha]_{\frac{20^{0}}{4^{0}}}=-32^{\circ} 2^{\prime} .}
\end{array}
$$

Der Ameisensäure-Ester des Fenchylalkohols siedet bei $13 \mathrm{Mm}$. bei $84^{0}-85^{\circ}, d_{15^{\circ}}=0,9880, \alpha_{D}=73^{\circ} 14^{\prime}$ (Bertram und Belle.)

\section{Isofenchylessigsäureester.}

Seinen physikalischen Eigenschaften nach ist dieser Ester dem Ameisensäureester sehr ähnlich, er unterscheidet sich von ihm durch seinen angenehmen aromatischen Geruch, ähnlich dem ätherischen Calmusöl.

Er siedet unter $12 \mathrm{Mm}$. Druck bei $97^{\circ}$.

Das spec. Gewicht betrug:

$$
d \frac{20^{\circ}}{20^{\circ}}=0,9676 . \quad d \frac{20^{\circ}}{4^{\circ}}=0,9659 .
$$

Die Bestimmung des Brechungsvermögens ergab:

$$
n_{D}=1,45719 \text {. }
$$

Molekularrefraction.

Berechnet für $\mathrm{C}_{10} \mathrm{H}_{18} \mathrm{CH}_{\mathrm{a}} \mathrm{COHO}$ :

Gefunden:

Nach Conrady's Zahlen

$$
55,00 \quad 55,17\left(20^{\circ}\right) ; 55,28\left(\frac{20^{\circ}}{4^{0}}\right) .
$$

Die Bestimmung des Drehungsvermögens ergab:

$$
\begin{array}{ll}
{[\alpha]_{D 20^{0}}=-18^{0} 11^{\prime} .} & \begin{array}{l}
\alpha_{D}=-17^{0} 36^{\prime} \\
{[\alpha]_{D_{20}}=-18^{0} 14^{\prime} .} \\
4^{\top}
\end{array}
\end{array}
$$


Kondakow: Synthesen unter Einw. v. Zinkchlorid etc. 229

Der Essigsäureisofenchylester, erhalten von Bertram und $\mathrm{Helle}$ aus dem Fenchen, siedet unter $8 \mathrm{Mm}$. bei $89^{\circ}$ bis $90^{\circ}$.

$$
d_{15}=0,9724 . . \quad \alpha_{D}=-14^{\circ} 46^{\prime} .
$$

Aus dem Isofenchylalkohol mit Essigsäureanhydrid gewonnen, siedet er bei $14 \mathrm{Mm}$. bei $98^{\circ}-99^{\circ}$.

$$
d_{15}=0,9740 . \quad \alpha_{D}=-10^{\circ} 58^{\prime} .
$$

Der Essigsäureester, aus dem Fenchylalkohol selbst nach der Methode von Bertram erbalten, hat folgende Eigenschaften.

Er siedet unter $10 \mathrm{Mm}$. bei $87^{\circ}$.

$$
d 19^{\circ}=0,9720 . \quad \alpha_{D}=-63^{\circ} 21^{\prime} .
$$

Erhalten aus dem Essigsäureanhydrid und Alkohol siedet er unter $10 \mathrm{Mm}$. bei $88^{\circ}$.

$$
d_{15}=0,9748 . \quad \alpha_{D}=-56^{\circ} 37^{\prime} . \quad[\alpha]_{D}=-58^{\circ} 08^{\prime} .
$$

Nach dem Verfahren von Bouchardat und Lafont aus dem Terpentinöl ${ }^{1}$ ) gewonnen, siedet er unter $50 \mathrm{Mm}$. bei $125^{\circ}-127^{\circ}$.

$$
d_{0^{0}}=0,9817 . \quad[\alpha]_{D}=+56^{\circ} 39^{\prime} .
$$

\section{Isofenchylvaleriansäureester.}

Erhalten aus dem Fenchen mit dem Drehungsvermögen $[\alpha]_{D}=+6^{0} 55^{\prime}$ und der Baldriansäure vom spec. Drehungsvermögen $[\alpha]_{D}=+2^{0} 8^{\prime}$. Der Geruch dieses Esters erinnert an Baldrian und Campher. Er hat den Siedepunkt von $142^{\circ}$ bis $145^{\circ}$ bei $19 \mathrm{Mm}$.

Die Bestimmung des Brechungsvermögens ergab:

$$
[\alpha]_{D}=1,45749 \text {. }
$$

Das spec. Gewicht betrug:

$$
d \frac{20^{\circ}}{20^{\circ}}=0,9437 \text {. }
$$

Molekularrefraction.

Berechnet für $\mathrm{C}_{10} \mathrm{H}_{16} \mathrm{C}_{5} \mathrm{H}_{10} \mathrm{O}_{2}$ :

Gefunden:

Nach Conrady's Zahlen

68,80

$68,73\left(20^{\circ}\right)$

Die Bestimmung des Drehungsvermögens ergab:

$$
\alpha_{D}=-30^{\prime} \text {. }
$$

Bei der Verseifung der obenbeschriebenen Ester des Isofenchylalkohols wird ein Alkohol erhalten wie bei Bertram

1) Compt. rend. 126, 756 (1898). 
230 Kondakow: Synthesen unter Einw. v. Zinkchlorid etc.

und Helle mit dem Schmelzpunkt $61,5^{\circ}$, aber mit anderen Drehungsvermögen, wie aus folgenden Angaben ersichtlich.

$s=0,0813 ; \quad L$ (Alkohol) $=2,949 ; \quad p=2,68 ; \quad d=0,796 ; \quad t^{\circ}=20^{\circ} ;$
$l=50 \mathrm{Cm} . ; \quad \alpha_{D}=+30^{\circ} ; \quad[\alpha]_{D}=+45^{\circ} 40^{\prime}$.

Auf solche Weise kann man nach den oben angeführten Thatsachen zu dem Schluss gelangen, dass das käufliche Camphen aus einem Gemisch ron zwei Isomeren besteht; eins derselben verbindet sich mit organischen Säuren unter oben beschriebenen Bedingungen und ergiebt eine theoretische Ausbeute an Ester des Isobornylalkohols, das andere Isomere, welches in sehr geringer Menge im Rohcamphen enthalten ist, bildet nicht solche Ester.

Das Rohfenchen besteht, ähnlich wie das Camphen, gleichfalls aus zwei Kohlenwasserstoffen, von denen der eine sich mit organischen Säuren verbindet und sich zu Estern des Isofenchylalkohols umwandelt; der andere verbindet sich nicht mit Säuren. Das letzte Isomere ist im Rohfenchen in so bedeutenden Mengen enthalten, dass es uns gelungen ist, es zu isoliren und ganz bestimmt $\mathrm{zu}$ charakterisiren.

Es fragt sich jetzt, welcher Art ist die Constitution dieser Isomeren des Camphens und Fenchens, welche die Eister des Isobornyl- und Isofenchylalkohols geben?

Diese Frage wird durch folgende einfache Annahmen erklärt. Die Thatsache, dass das Camphen und Fenchen, indem sie bei obenbeschriebenen Bedingungen organische Säuren addiren, ähnlich reagirend wie die Aethylenkohlenwasserstoffe, welche bei der Doppelbindung ein primär-tertiäres, secundärtertiäres und tertiär-tertiäres Kohlenstoffatom besitzen, giebt uns Grund anzunehmen, dass sich in den beiden obenerwähnten Kohlenwasserstoffen eine Doppelbindung von ähnlichem Charakter wie bei dem tertiären Kohlenstoffatom befinden muss.

Eine derartige Doppelbindung kann sich bei ihnen entweder im Ringe, natürlich mit Ausnahme der primär-tertiären, oder aber in der Seitenkette befinden.

Die Annahme der Gegenwart einer Doppelbindung im Ringe bei diesen Kohlenwasserstoffen schliesst sich aus folgenden Erwägungen aus. Wenn solche Kohlenwasserstoffe, wie das Tetrahydrotoluol, Menthomenthen und Carvomenthen nicht Ester nach meinem Verfahren geben, aber aus dem 
Kondakow: Synthesen unter Einw. v. Zinkchlorid etc. 231

Camphen und Fenchen solche erhalten werden, so bedeutet das, dass der Charakter und die Lage der Doppelbindung in diesen Kohlenwasserstoffen verschieden ist, und wenn in den ersteren drei die Doppelbindung zweifellos sich im Ringe befindet, so muss sie sich in beiden letzten in der Seitenkette befinden.

Diese Annahme der Gegenwart einer Doppelbindung bei der Seitenkette, den Isomeren des Camphens und Fenchens, welche leicht organische Säuren unter Einwirkung von Zinkchlorid addiren, folgt nicht nur aus der Aehnlichkeit dieser Kohlenwasserstoffe, wie das Isobutylen und das asymmetrische Methyläthyläthylen zu reagiren, aus der Unähnlichkeit mit den anderen hydroaromatischen Kohlenwasserstoffen, sondern auch daraus, dass das Dipenten unter denselben Bedingungen, wie es Schindelmeiser in unserem Laboratorium bewies, mit organischen Säuren Derivate giebt, die dem Terpineol und den Produkten entsprechen, welche sich auf Kosten der Doppelbindung in der Seitenkette aber nicht im Ringe bilden.

Weiter wird diese Folgerung noch dadurch berechtigt dass nach dem Verseifen der nach meinem Verfahren dargestellten Ester aus Camphen der tertiäre Alkohol Isoborneol erhalten wird. Folglich konnten seine Ester sich nur auf Kosten desjenigen Kohlenwasserstoffs bilden, welcher wenigstens ein tertiäres Kohlenwasserstoffatom bei der Doppelbindung besitzt.

Da aber ein solches Kohlenwasserstoffatom sich weder in der Constitutionsformel von Bredt noch in der von Tiemann befindet, so bleibt nur noch eins übrig, sie für nicht entsprechend (nicht richtig) anzunehmen. Die oben angeführten Erwägungen berücksichtigend, könnte man die Constitutionsformel des Camphens nach Bredt oder Tiemann derart umändern, dass man die Doppelbindung zur Diagonalbindung so verlegt, dass in ibr das tertiäre Kohlenstoffatom bei der Doppelbindung im Ringe läge.

Aber eine solche Lage der Doppelbindung stimmt nicht mit den obigen Erwägungen überein, ebenso nicht mit den vielfachen thatsächlichen Ergebnissen, welche bei der Oxydation dieses Kohlenwasserstoffs erhalten wurden.

Es bleibt daher nach allen Erwägungen nur übrig, in dem käuflichen Camphen ein Isomeres mit einer Doppelbindung an einem tertiären Koblenstoffatom nur in der 
232 Kondakow: Synthesen unter Einw. v. Zinkchlorid etc.

Seitenkette anzunehmen und es für ganz anders constituirt anzusehen, als es bisher angenommen wurde. Man kann aber auch zulassen, dass die Doppelbindung sich in diesem Kohlenwasserstoff im Ringe befindet und nur seine Eigenschaften zu Gunsten der leichten Reactionsfähigkeit mit organischen Säuren in der Richtung der Diagonalbindung verändert. Dieser Zulassung steht im Wege, dass das Pinen, welches ausser der Doppelbindung im Ringe noch eine Diagonalbindung besitzt, nach meinem Verfahren keine Derivate giebt, welche sich auf Kosten der Doppelbindung bilden (das Pseudopinen gelang es uns nicht im Pinen nach meinem Verfahren za finden), - aber nur solche Produkte, welche bei der Auflösung der Diagonalbindung (Piceanring) nach verschiedener Richtung entstehen. ${ }^{1}$ )

1) Der Piceanring kann sich nach zwei Richtungen auflösen. Indem sie sich nach der einen von ihnen auflöst, giebt sie Derivate des Terpineols, welche durch innere Condensation, in Uebereinstimmung mit den bestehenden Erklärungen, sich in Borneolderivate umwandeln.

Die Auflösungsrichtung der Diagonalbindung des Pinens ist aber nicht die einzige, da durch sie es gar nicht möglich ist, die Bildung der Derivate des Fenchylalkohols aus dem Pinen zu erklären, ohne complicirte Umwandlungen zuzulassen oder ohne eine Beimengung von Fenchen anzunehmen.

Nach meiner Ansicht kann man die Entstehung der Fenchylderivate aus dem Pinen unter der Einwirkung der Benzoësäure, wie es Bouchardat und Lafont (Compt. rend. 113, 531; 126, 755) zeigten, unter der Einwirkung von Trichloressigsäure nach Reychler, und endlich der Monochloressigsäure und Essigsäure in Gegenwart von Zinkchlorid nach meinem Verfahren, wie es in meinem Laboratorium Schindelmeiser zeigte, ganz einfach ohne Zulassungen complicirter Umwandlungen erklären, eben auf folgende Weise. Das Pinen, inderr es ein Molekül Săure unter oben angeführten Bedingungen addirt, giebt folgende intermediäre Verbindung<smiles>[X]C(C)(C)C1CCCCC1</smiles>

welche nach der Addition eines zweiten Moleküls der Säure jetzt an der Stelle der Doppelbindung in folgende zwei Produkte übergeht: 
Kondakow: Synthesen unter Einw. v. Zinkchlorid etc. 233

Die Frage aber über die Lage der Doppelbindung selbst im Camphen wird ihrerseits durch folgende Erwägung aufgeklärt.

Die Entstehung des Camphens aus den Derivaten des tertiären Isobornylalkohols nöthigt uns, es für ein directes Derivat dieses Alkohols anzusehen, welches sich aus ihm ohne jede Umlagerung bildet. Die oben bewiesene Unmöglichkeit der Gegenwart einer Doppelbindung im Ringe des Camphens zwingt uns anzunehmen, dass am Verlust der Wasserelemente (oder der entsprechenden Gruppen, wenn es sich um die Derivate dieses Alkohols handelt) aus dem Isobornylalkohol eine der Seitenkette theilnimmt, welche sich dabei an dem Kohlenstoffatom befindet, an welchen die Hydroxylgruppe gebunden ist.

Aus den angeführten verschiedenen theoretisch möglichen Constitutionsformeln des Camphens, entsprechend solchen Anforderungen, und ebenso aus allen Umwandlungen sowohl seiner selbst als auch den Umwandlungen des Camphers und Borneols würde am meisten die Constitutionsformel des Camphens befriedigen, welche zuerst von Tiemann vorgeschlagen wurde, aber
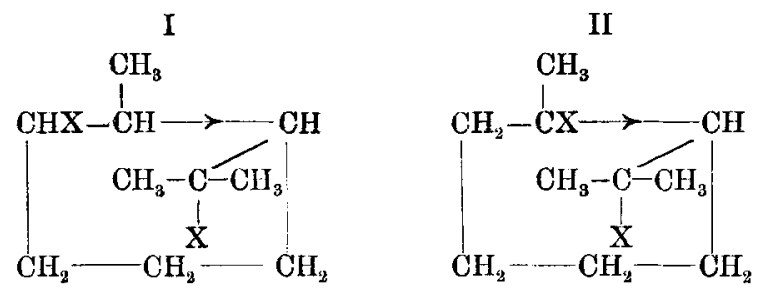

Diese beiden Verbindungen geben nach der Abspaltung eines Moleküls Säure entweder Derivate des Fenchyl- oder Isofenchylalkohols:
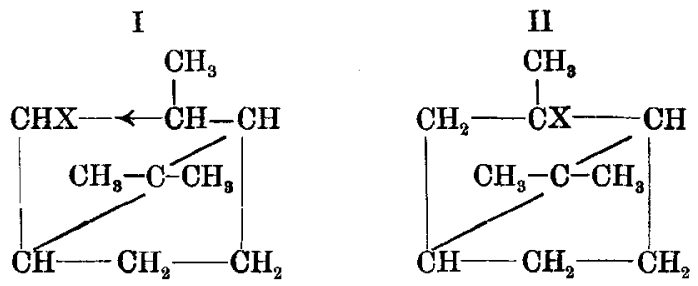

Diese Erklärung ist durchaus nicht hypothetischer, als die Erklärung von Wagner und Semmler über die Entstehung der Bornylderivate aus dem Pinen. 
234 Kondakow: Synthesen unter Einw. v. Zinkchlorid etc.

nur mit der Umänderung, die in ihr von Wagner gemacht wurde, wenn es nur erwiesen sein würde, dass die Seitenketten im Camphene sich in der Orthostellung zu einander befinden und dass bei der Auflösung der vorausgesetzten Diagonalbindung sich trisubstituirte Derivate des Hexahydrobenzols bilden.

Da aber directe Ergebnisse fehlen, so bleibt nur übrig, die Constitutionsformel des Camphens von Borneol oder Campher abzuleiten, wobei in den letzten beiden Verbindungen bestimmte Umwandlungen zugelassen werden müssen; in Uebereinstimmung damit müssen die von verschiedenen Forschern für das Camphen vorgeschlagenen Formeln umgeändert werden.

Nachdem auf diese Weise festgestellt war, dass das käufliche Camphen fast ausschliesslich aus dem Isomeren mit der Doppelbindung in der Seitenkette besteht, was ganz mit der Bildung des Camphenylons (Wagner) bei der Oxydation des Camphens mit dem Permanganat übereinstimmt, ist noch aufzuklären, welche Constitution dem anderem Isomeren des Camphens zukommt, welches in sehr geringen Mengen in dem käuflichen Camphen enthalten ist.

Die Unfähigkeit dieses Camphens, sich unter oben angeführten Bedingungen mit organischen Säuren zu verbinden, weist auf seine zweifellose Aehnlichkeit einerseits mit den Aethylenkohlenwasserstoffen mit secundär-secundärem und primär-secundärem Kohlenstoffatom bei der Doppelbindung hin, anderseits aber auch auf das Tetrahydrotoluol, Menthomethen und Carvomenthen; sie giebt uns auch das Recht anzunehmen, dass dieses zweite Isomere des Camphens die Doppelbindung im Ringe bei dem secundär-secundären Kohlenstoffatom enthält und das echte Derivat des Borneols vorstellt.

Dieser Schluss wird durch folgendes bestätigt: es ist bekannt, dass das Gemisch von Camphen und Isocamphen, welches von Wagner aus dem Jodwasserstoffpinen ${ }^{1}$ ) (Bornyljodid?)

1) Das Verhalten des Jodwasserstoffpinens zu starker alkoholischer Kalilauge, welches eine nur geringe Menge Camphen (Bornylen) giebt, genügt nicht zur Behauptung, dass dieses Jodid reines Bornyljodid ist. Es drängt sich von selbst die Frage auf, warum sich nicht das ganze Jodid zu. Camphen umwandelt, sondern nur ein Theil desselben. Diese Thatsache kann man entweder dadurch erklären, dass dieses Jodid kein 
Kondakow: Synthesen unter Einw. v. Zinkchlorid etc. 235

erhalten wurde, bei der Bearbeitung mit Essigsäure in Gegenwart von Schwefelsäure nach der Methode von Bertram und Walbaum ${ }^{1}$, wie es Wagner zeigte, sich so weit mit der Essigsäure verbindet, als in ihm das Isocamphen (Isobornylen) enthalten ist, das Camphen (Bornylen) tritt nicht in Reaction. ${ }^{2}$ ) Dasselbe findet auch augenscheinlich bei unserer Methode, bei der Esterification der hydroaromatischen Kohlenwasserstoffe statt, da sie dem Verfahren von Bertram und Walbaum ähnlich ist, wie es in unserem Laboratorium von Herrn Schindelmeiser durch Vergleich dieser Methoden untereinander festgestellt worden ist.

Zur Frage über die Constitution des Fenchens übergehend, muss bemerkt werden, dass alles oben über das Camphen Gesagte im gleichen Maase auf diesen Kohlenwasserstoff bezogen werden kann, nur mit dem Unterschiede, dass die Frage hier einfacher entschieden wird.

Wenn wir die Constitution des Fenchylalkohols, wie sie $\mathrm{ihm}$ von $\mathrm{W}$ allach gegeben worden ist, für richtig anerkennen, woran zu zweifeln kein Grund vorliegt, so wird eines der Fenchene diesem Alkohol entsprechen und die Doppelbindung im Ringe enthalten. Dieser Kohlenwasserstoff wird den Menthenen

secundäres, kein Bornyljodid ist, oder dadurch, dass es als secundäres fast ganz während der Reaction in tertiäres isomerisirt wird, oder auch endlich damit, dass es aus einem Gemisch von secundärem und tertiärem Isomeren besteht.

Wenn es ein secundäres Jodid ist, so isomerisirt sich in solchem Fall das aus ihm entstandene anfängliche Camphen unter der Einwirkung der starken alkoholischen Lauge zu Isocamphen. Wenn es ein tertiüres ist, so kann das Umgekehrte eintreten, d. h. das Isocamphen isomerisirt sich theilweise zum Camphen. Das ungleiche Verhalten dieses Jodids zum $\mathrm{Ka}$ liumphenolat und zu starken alkoholischen Alkalilaugen unterstïtzen die oben ausgesprochene Folgerung, theilweise auch die letztere von ihnen über die mögliche Isomerisation des Isocamphens unter der Einwirkung der lang dauernd siedenden alkoholischen Alkalilauge zum Camphen. Dafür, dass eine solche Isomerisation möglich ist, hat man einige Thatsachen, unter anderem nach unseren Untersuchungen ïber das Fenchen. Zur Ausscheidung derartiger Zweifel sind natürlich entsprechende Versuche nöthig, welche in den Versuchen von Wagner fehlen.

1) Dies. Journ. [2] 41, 1.

2) Ber. 33, $2122(1900)$. 
236 Kondakow: Synthesen unter Einw. v. Zinkchlorid etc.

am ähnlichsten sein und sich wie dieses in Gegenwart von Zinkchlorid nicht mit organischen Säuren verbinden.

Ein Kohlenwasserstoff mit gerade solchen Eigenschaften wurde von uns bei der Bearbeitung des Rohfenchens nach unserem Verfahren isolirt.

Dieser Kohlenwasserstoff entspricht zweifellos dem Camphen von Tschugaeff, er unterscheidet sich indessen erstens von ihm durch die Anwesenheit des tertiären Kohlenstoffatoms bei der Doppelbindung und zweitens wird er ein Derivat des Metacymols sein.

Die Constitution dieses Fenchens wurde zuerst von $W$ allach und in allerletzter Zeit auch von ihm durch Ergänzungsversuche bestätigt. Die Oxydation dieses Fenchens wird jetzt bei uns ausgeführt. Das andere Isomere des Fenchens, dessen Bildung aus dem Fenchylalkohol anormal ist und von Niemandem vor uns vorausgesehen war, entspricht dem Iso-Camphen (Isobornylen) und hat die Constitution, welche ihm von uns zuerst gegeben worden ist.

Dieses zweite Fenchen, welches dem Isofenchylalkohol entspricht, bildet sich aus ihm (oder aus seinen Haloidanhydriden) durch Verlust der Elemente des Wassers unter Theilnahme der Methylgruppe.

Folglich hat dieses Fenchen die Doppelbindung in der Seitenkette bei dem primär-tertiären Kohlenstoffatom und ist dem Isocamphen, den Olefinen und hydroaromatischen Kohlenwasserstoffen gleicher Constitution ähnlich. Bei der Esterification des Rohfenchens nach unserer Methode bildet sich nämlich aus diesem Isomeren der Ester des Isofenchylalkohols. Solch eine Folgerung stimmt mit der Bildung des Fenchocamphorons bei der Oxydation des Fenchens mit Permanganat, welche von Wallach constatirt ist, überein.

Die Frage, ob im Rohfenchen noch ein drittes Isomeres enthalten ist, wie es Wallach annimmt, unterliegt noch

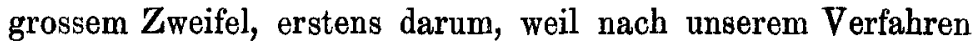
dieses Isomere nicht entdeckt werden konnte, zweitens auch darum, weil die Thatsachen, welche W allach zu solcher Schlussfolgerung brachten, nicht genug überzeugend sind. Jedenfalls ist diese Frage bis auf Weiteres offen. 
Kondakow: Synthesen unter Einw. v. Zinkchlorid etc. 237

Aus der Gesammtheit aller oben angeführten Thatsachen und Erwägungen ergeben sich folgende Schlüsse:

1. Die von mir ausgesprochene Behauptung, dass "das Zinkchlorid zur Entdeckung des tertiären Kohlenstoffatoms bei Doppelbindung dienen kann ...." hat sich als durchaus richtig erwiesen, nur mit der Beschränkung, dass eine derartige Doppelbindung in den hydroaromatischen Verbindungen nur dann entdeckt wird, wenn sie sich in der Seitenkette aber nicht im Ringe befindet.

2. Die oben von mir beschriebene Methode der Esterification hydroaromatischer Kohlenwassertoffe mit der Aethylenbindung in der Seitenkette ist besser als die Methode von Bertram und Walbaum, wie in unserem Laboratorium durch Vergleichsversuche festgestellt wurde.

3. Das von mir vorgeschlagene Verfahren gab die Möglichkeit zu beweisen, dass das käufliche Camphen und das Fenchen aus zwei chemischen Isomeren bestehen, von denen das eine die Doppelbildung in der Seitenkette, das andere in dem Ringe besitzt.

Die Verschiedenheit in der Reactionsfähigkeit der beiden Isomeren des Fenchens gab die Möglichkeit, eines derselben in reiner Form zu isoliren. Dasselbe Verfahren kann auch zweifellos zur Trennung der Camphene dienen. ${ }^{1}$ )

4. Bei der Umwandlung secundärer hydroaromatischer Alkohole in entsprechende Haloïdanhydride nach bekannten Methoden, wird, wie wir feststellten, ein Gemenge secundärer Derivate mit tertiären erhalten, letztere vorherrschend. Ein derartiges Gemenge von Halö̈anhydriden giebt beim Ab-

1) In der Nr. 13 der Ber, d. J. S. 3283 beschreibt Zelinsky zwei Camphene aus 1. und r-Borneol als flüssige Kohlenwasserstoffe. Zelin = sky's Camphene krystallisiren darum nicht, weil sie Beimengung von Isomerisationsprodukten (von Fenchen oder Pinen?), welche sich auf Kosten der entstandenen Ameisensäure gebildet haben, enthalten. Nebenbei gesagt, ist es uns viel früher als Zelinsky beim Bearbeiten des rBorneol $[\alpha]_{D}=33^{\circ} 44$ mit entwässerter Oxalsäure gelungen, neben flüssigem Camphen noch eine gesättigte optisch active $+38^{\circ} 26^{\prime}$ krystallinische Verbindung von dem Schmelzp. 105,50 zu gewinnen. Dabei möchten wir Hrn. Zelinsky in Erinnerung rufen, dass die Oxalsäure von uns zur Darstellung des 'Trimethyläthylens (dies. Journ. [2] 54, 485) und des Pinakolins aus dem Pinakon (Chem. Zeit. 1899, Nr. 29) angewandt wurde. 
238 Kondak ow: Synthesen unter Einw. v. Zinkchlorid ete.

spalten der Haloidwasserstoffsäuren nach bekannten Regeln natürlich ein Gemisch von Kohlenwasserstoffen, welche jedem der Haloïdanhydride entsprechen. Die tertiären Haloïdanhydride, welche durch Isomerisation der secundären, oder durch die intermediären Bildungsstufen von Kohlenwasserstoffen, oder durch die Umlagerung der Atome im Molekül entstehen, verlieren leichter die Haloüdwasserstoff'säuren, als die secundären.

5. Die von mir entdeckte Synthese in der Fettreihe unter Einwirkung von Zinkchlorid ergab ihre Anwendbarkeit in der hydroaromatischen Reihe.

Die Methode für Synthesen hat eine ebenso theoretische als auch praktische Bedeutung wie die Synthesen nach Friedel und $\mathrm{Crafts}$; die Fälle, in welchen sie anwendbar ist, erwiesen sich als sehr zahlreich.

Zur Zeit ist es mir gelungen, zu beobachten, dass die hydroaromatischen Verbindungen mit verschiedenen Functionen, welche eine Doppelbindung im Ringe und in der Seitenkette haben, aromatische Verbindungen mit ungesättigter Seitenkette und endlich Verbindungen der Fettreihe mit vielfacher Bindung in Gegenwart von Zinkchlorid mit Säuren, mit Chloranhydriden und Säureanhydriden, mit Haloidanhydriden der Alkohole reagiren.

Die Einzeluntersuchung der zahlreichen Verbindungen, welche dabei erhalten werden, verspricht nicht wenige neue und interessante Ergebnisse. Ich hoffe, dass die Forscher die von mir vorgeschlagene Methode nicht ohne entsprechende Berücksichtigung lassen werden. In der nächstfolgenden $\mathrm{Ab}$ handlung werden Thatsachen mitgetheilt werden, welche sich auf die nach meinem Verfahren ausgeführte Esterification von hydroaromatischen Kohlenwasserstoffen mit zwei Doppelbindungen beziehen. An dem experimentellen Theil betheiligten sich die Herren Schindelmeiser und Lutschinin; ich halte es für eine angenehme Pflicht, ihnen meinen Dank auszusprechen.

Dorpat (Jurjew), den 21. Oct./3. Nov. 1901. 